RESEARCH ARTICLE

\title{
Abiotrophia and Granulicatella Infections in Cancer Patients: A Single- Center Chart Review Study
}

\author{
Jooeun Kwon ${ }^{1}$, Dae Hyun Lee ${ }^{2}$, George Coba ${ }^{1}$, Annie Topham ${ }^{3}$, Misbahuddin Syed ${ }^{4}$, Asha Ramsakal $^{5}$, \\ John Greene ${ }^{6}$ \\ ${ }^{1}$ Morsani College of Medicine, University of South Florida, Tampa, FL, USA \\ ${ }^{2}$ Division of Cardiovascular Science, Department of General Internal Medicine, Morsani College of Medicine, University of \\ South Florida, Tampa, FL, USA \\ ${ }^{3}$ Department of General Internal Medicine, Morsani College of Medicine, University of South Florida, Tampa, FL, USA \\ ${ }^{4}$ Internal and Hospital Medicine, H. Lee Moffitt Cancer Center and Research Institute, Tampa, FL, USA \\ ${ }^{5}$ Department of Internal Medicine, H. Lee Moffitt Cancer Center and Research Institute, Tampa, FL, USA \\ ${ }^{6}$ Infectious Diseases and Hospital Epidemiologist, H. Lee Moffitt Cancer Center and Research Institute, Tampa, FL, USA
}

\section{ABSTRACT}

Objectives: Nutritionally Variant Streptococci (NVS) comprise two primary genera that have been identified from human specimens, particularly the Abiotrophia and Granulicatella species. These infections are often complicated to manage due to the difficulty identifying these organisms, indolent course of disease, and variable resistance to common antimicrobial agents. The wide breadth of disease presentations involving these organisms has not been fully elucidated, particularly in the immunosuppressed cancer patient population.

Method: We performed a retrospective chart review on 28 patients from an academic cancer center with positive NVS cultures, from January 2012 to July 2018. We reviewed patient characteristics, culture data, immunodeficiency status, response to antibiotic therapy, and outcomes of infection.

Results: Of twenty-eight patients, fifteen patients developed bacteremia from either Abiotrophia or Granulicatella species, while thirteen patients had positive wound or body fluid cultures. Most patients with bacteremia had underlying hematologic malignancies and neutropenia. Patients with positive wound or body fluid cultures had an invasive procedure at the related site. Intravenous (IV) vancomycin was the most common agent used, and all but two patients were treated with multiple antibiotic regimens.

Conclusions: Infections with NVS have been reported with a variety of clinically infectious presentations and should be considered in cancer patients with neutropenia or in patients who have undergone invasive procedures. Bacteremia was the most common complication, especially in the setting of hematologic malignancy and neutropenia. Focal body site infection was also a common complication related to invasive procedures in immunocompetent patients. Overall mortality was low and related to complications of septic shock. J Microbiol Infect Dis 2020; 10(2):89-97.

Keywords: Abiotrophia, Granulicatella, bacteremia, cancer, neutropenic infection

\section{INTRODUCTION}

Nutritionally Variant Streptococci (NVS) was first described by Frenkel and Hirsch in 1961 as a New Viridans Group Streptococci (VGS) that form satellite colonies around other bacteria [1].
These bacteria depend on other bacteria or enriched media as they do not synthesize pyridoxine, L-cysteine, or other essential nutrients required for growth. Like other VGS, Abiotrophia and Granulicatella species are part of the normal flora of the oral cavity, urogenital,

Correspondence: Dr. John Greene, H. Lee Moffitt Cancer Center and Research Institute,

12902 Magnolia Drive, FOB-3, Tampa, FL, USA

Email: John.Greene@moffitt.org

Received: 23 November 2019 Accepted: 30 May 2020

Copyright (C JMID / Journal of Microbiology and Infectious Diseases 2020, All rights reserved 
and intestinal tracts [2]. These species are implicated in a variety of clinical infections, the most common being bacteremia and infective endocarditis [3]. Despite being rare, Abiotrophia infective endocarditis is associated with increased mortality and serious complications such as rapid valve destruction, heart failure, and embolic events [4,5]. Isolated cases of central nervous system infections [6] and septic arthritis [7] have also been reported. These organisms can cause severe infections in both immunocompetent and immunosuppressed hosts $[5,8]$.

Identifying Abiotrophia and Granulicatella have been challenging in the past due to their fastidious nature [1,2]. However, with improvement of culture media, these organisms can now be detected in routine blood cultures in 2-3 days. However, there are still challenges to accurately speciating Abiotrophia and Granulicatella species [9]. In addition, the clinical course can be unfavorable despite the administration of the recommended antibiotic regimens [10]. We aimed to identify and analyze all cases of Abiotrophia and Granulicatella infections at an academic cancer center from January 2012 to July 2018 to better characterize the presentation and susceptibility of Abiotrophia and Granulicatella in patients with underlying malignancy.

\section{METHODS}

We retrospectively reviewed all cases of positive Abiotrophia or Granulicatella cultures occurring from January 2012 to July 2018 at H. Lee Moffitt Cancer Center and Research Institute. The computerized epidemiology report provided by the microbiology laboratory identified 28 patients with positive Abiotrophia or Granulicatella cultures. Positive isolates were identified by the presence of a satellite using the Pyridoxal Disk. Further speciation to distinguish Granulicatella and Abiotrophia species was done using the VITEK 2 system upon request by physician. Susceptibility testing was done upon request through ARUP Laboratory in Salt Lake City, Utah.

Variables observed included patient demographics such as age and sex, clinical symptoms, underlying malignancy, immune status, culture, immunosuppressant medications, treatment of infection, and outcome of infection. Data was recorded from the Infectious Disease consultation reports, discharge summaries, lab results, cultures, and medication profiles using Power Chart/Cerner at Moffitt Cancer Center. Resolution of the infection was defined as repeated negative blood cultures and clinical resolution of signs and symptoms of infected sites. Quantitative description was used for summarizing the data. Microsoft Excel (California, USA) was used to summarize data in mean $( \pm$ standard deviation) or number (percentage). A table containing these data was made and analyzed for any trends or details.

Informed consent was not necessary due to the retrospective nature of this work and deidentification of data collected from chart review. The methods of this study were approved by the University of South Florida Institutional Review Board (Approval \#Pro00034405). The study has been performed in accordance with the 1964 Declaration of Helsinki and its lateral amendments.

\section{RESULTS}

\section{Overall Characteristics}

There were 28 patients with cultures positive for Abiotrophia or Granulicatella species during the study period. The mean age of patients affected was 63.7 years, of which 15 (54\%) were male, and $13(46 \%)$ were female. Hematologic cancers, particularly AML, were the most common underlying malignancy. Colon cancer was the most common solid tumor malignancy. More details of the underlying malignancies are listed in Table 1. There were fifteen positive blood cultures and thirteen positive wound or other body fluid cultures as shown in Figure 1.

\section{Characteristics and Treatment of Bacteremia}

The clinical presentation and treatments used in patients with bacteremia are shown in Table 2 . Fever was the most common clinical presentation, followed by gastrointestinal symptoms (abdominal pain) and mucositis. Most patients with bacteremia had an underlying hematologic malignancy (87\%) and most were 
neutropenic $(73 \%)$. The average duration of neutropenia at the time of positive culture was 14.3 days (neutropenia was defined as ANC $<500 \mathrm{cell} / \mathrm{mm} 3$ ). Twelve patients were on corticosteroids, chemotherapy agents, or other medications that may have contributed to their immunocompromised state which are further detailed in Table 2. The most common agents for empiric antibiotic therapy prior to culture results were ciprofloxacin, cefepime, and piperacillin-tazobactam. All patients with bacteremia were ultimately treated with multiple antibiotic regimens (average 3.2), with most common being vancomycin IV (average duration 6.8 days) and cefepime IV (average duration of 4.2 days.). Thirteen patients with bacteremia survived (87\%), and the resolution of infection was confirmed by repeat negative blood cultures and clinical resolution of signs and symptoms. Two patients with bacteremia expired due to complications related to septic shock.

\section{Characteristics and Treatment of Focal Body Site Infections}

The clinical presentation and treatments used in patients with wound or body fluid positive cultures are shown in Table 3. Most wound/body fluid positive cultures (85\%) were related to a procedure site or recent surgical site, and cultures were ordered due to nonspecific symptoms of infected wound, including fever and redness or drainage from wound site. Two patients had no significant symptoms and had cultures collected during an operation. The most common prophylactic or prior empiric antibiotic therapy agents were cefepime, ceftriaxone, or cefoxitin. Eleven patients were treated with multiple antibiotic regimens (average 2.3), with the most common being cefepime IV (average duration 12.8 days), ciprofloxacin PO (average duration 7 days), or vancomycin IV (average duration 5 days). Twelve patients survived with resolution of infection, while one patient with positive $G$. elegans urine culture expired due to complications related to septic shock.

\section{Susceptibility}

Susceptibility report showed that NVS species were most susceptible to vancomycin and meropenem, with variable susceptibility to penicillin G, ceftriaxone, cefepime, levofloxacin, and ceftazidime. There was resistance to clindamycin and erythromycin, as further detailed in Table 4.

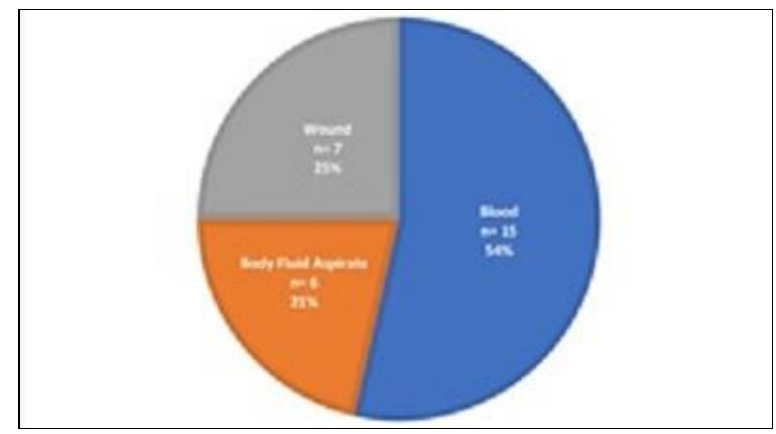

Figure 1. The Sources of isolated bacteria.

Table 1. Underlying malignancy of the cases.

\begin{tabular}{lc}
\hline Primary Diagnosis & No. Cases (\%) \\
\hline Hematologic & $14(50 \%)$ \\
AML & $11(39 \%)$ \\
\multicolumn{1}{c}{ MDS } & $2(7 \%)$ \\
Adenocarcinoma of esophagus & $1(3.5 \%)$ \\
Breast cancer & $1(3.5 \%)$ \\
Cholangiocarcinoma & $1(3.5 \%)$ \\
Colon Cancer & $1(3.5 \%)$ \\
GIST & \\
Lipoma & $3(11 \%)$ \\
Melanoma & $1(3.5 \%)$ \\
Osteosarcoma & $1(3.5 \%)$ \\
Renal cell carcinoma & $1(3.5 \%)$ \\
Squamous cell carcinoma of RLL & $1(3.5 \%)$ \\
Urothelial carcinoma & $2(7 \%)$ \\
\hline AMLAcut & $1(3.5 \%)$ \\
\hline
\end{tabular}

AML: Acute Myeloid Leukemia

ALL: Acute Lymphoblastic Leukemia

MDS: Myelodysplastic Syndrome

GIST: Gastrointestinal Stromal Tumor 
Table 2. Characteristics of the patients diagnosed with bacteremia.

\begin{tabular}{|c|c|c|c|c|c|c|c|c|}
\hline Case & $\begin{array}{l}\text { Underlying } \\
\text { Malignancy }\end{array}$ & $\begin{array}{l}\text { Underlying } \\
\text { Cardiac } \\
\text { Conditions }\end{array}$ & $\begin{array}{c}\text { Chemotherapy/ } \\
\text { immunosuppressive } \\
\text { therapy }\end{array}$ & $\begin{array}{c}\text { Days of Neutropenia }(A N C< \\
500 \text { cells } / \mathrm{mm} 3)\end{array}$ & $\begin{array}{l}\text { Presentation/ } \\
\text { Clinical } \\
\text { Symptoms }\end{array}$ & Treatment & Isolation & Outcome \\
\hline 1 & AML & none & \multirow{2}{*}{$\begin{array}{l}\text { CYT, idarubicin, } \\
\text { etoposide/DXM } \\
\text { Idarubicin }\end{array}$} & 5 & \multirow{3}{*}{$\begin{array}{c}F, \text { pain and swelling } \\
\text { to tooth extraction site } \\
F \text {, mucositis } \\
F \text {, abdominal pain, } \\
\text { jaundice } \\
F \text {, nausea }\end{array}$} & \multirow{3}{*}{$\begin{array}{c}\text { CFX IV }^{8} 7 \mathrm{~d}, \text { VA IV n/a, } \\
\text { PTZ IV n/a } \\
\text { VA IV n/a, CEP IV n/a } \\
\text { CFX IV 2d, MER IV } 5 \mathrm{~d} \\
\text { ERT IV } 15 \mathrm{~d}\end{array}$} & $N V S^{6}$ & SR \\
\hline 2 & APML & none & & 15 & & & NVS & SR \\
\hline 3 & Colon cancer & none & Fluorouracil & 1 & & & $N V S$ & $\mathrm{SR}$ \\
\hline 4 & ALL & none & $\begin{array}{l}\text { Fludarabine, Busulfan } \\
\text { Etoposide, } \\
\text { Methotrexate/ DXM }\end{array}$ & 21 & \multirow{3}{*}{$\begin{array}{l}\text { and vomiting, } \\
\text { mucositis, diarrhea } \\
\text { F, mucositis, hypotension, } \\
\text { altered mental status } \\
\text { F, mucositis, rash, } \\
\text { respiratory distress }\end{array}$} & $\begin{array}{c}\text { VA IV } 14 d, \text { CFX IV } 2 d \\
\text { LEV PO }^{9} 7 d\end{array}$ & NVS & SR \\
\hline 5 & AML & Atrial fibrillation & None & 14 & & CEP IV $4 \mathrm{~d}$, VA IV $11 \mathrm{~d}$ & NVS & EX \\
\hline 6 & AML & none & Idarubicin, CYT/ MP & $n / a^{10}$ & & VA IV $5 \mathrm{~d}$, CEP IV $5 \mathrm{~d}$ & $N V S$ & SR \\
\hline 7 & Renal mass & $\begin{array}{l}\text { Chronic heart } \\
\text { failure }\end{array}$ & \multirow{3}{*}{$\begin{array}{c}\text { None } \\
\text { Idarubicin, CYT } \\
\text { Cladribine/ MP, DXM } \\
\text { Idarubicin, CYT } \\
\text { hydrocortisone }\end{array}$} & 0 & $\mathrm{~F}$ & $\begin{array}{c}\text { VA IV } 9 \mathrm{~d}, \text { AMC PO } 5 \mathrm{~d} \\
\text { CEP PO } 5 \mathrm{~d}\end{array}$ & $\begin{array}{c}G . \\
\text { adiacens }\end{array}$ & SR \\
\hline 8 & AML & Heart murmur & & 7 & Gl bleed, productive cough & $\begin{array}{l}\text { LEV PO } 2 d, \text { MER IV } 6 d \\
\text { VA IV } 2 d\end{array}$ & & SR \\
\hline 9 & AML & none & & 3 & \multirow{5}{*}{$\begin{array}{c}\mathrm{F} \text {, fatigue, hematuria } \\
\mathrm{F} \text {, cellulitis (wrist), } \\
\text { abdominal } \\
\text { pain, chest pain } \\
\text { F, respiratory failure, rash, } \\
\text { weight loss, gingivitis/poor } \\
\text { dentition } \\
\mathrm{F} \text {, chest pain, abdominal } \\
\text { pain, } \\
\text { night sweats } \\
\mathrm{F}\end{array}$} & CLN IV 8d, VA 10d IV & $\begin{array}{l}G \text {. } \\
\text { adiacens }\end{array}$ & SR \\
\hline 10 & AML & none & CYT & 14 & & $\begin{array}{c}\text { VA IV } 14 \text { d, CEP IV } 2 \text { d } \\
\text { PTZ IV } 9 d\end{array}$ & $\begin{array}{c}G . \\
\text { adiacens }\end{array}$ & SR \\
\hline 11 & APML & none & \multirow{5}{*}{$\begin{array}{c}\text { CYT, idarubicin } \\
\text { Vincristine, cyclophosphamide } \\
\text { Dasatinib, } \\
\text { doxorubicin/ DXM } \\
\text { CYT, idarubicin, etoposide/ } \\
\text { DXM } \\
\text { Sirolimus, tacrolimus } \\
\text { MP, prednisone } \\
\text { Melphalan, cetuximab, } \\
\text { fluorouracil, } \\
\text { irinotecan/ sirolimus, DXM, } \\
\text { MP }\end{array}$} & 15 & & $\begin{array}{l}\text { VA IV } 14 \mathrm{~d} \text {, CIP PO } 2 \mathrm{~d} \\
\text { CEF IV } 8 \mathrm{~d}, \text { CEP IV } 4 \mathrm{~d}\end{array}$ & $\begin{array}{c}G . \\
\text { adiacens }\end{array}$ & SR \\
\hline 12 & ALL & none & & 21 & & $\begin{array}{c}\text { VA IV } 12 \mathrm{~d}, \text { CEP IV } 2 \mathrm{~d} \\
\text { MER IV } 4 \mathrm{~d}\end{array}$ & $\begin{array}{c}G . \\
\text { adiacens }\end{array}$ & SR \\
\hline 13 & AML & none & & 0 & & $\begin{array}{l}\text { VA IV n/a, DOX } \\
\text { intrapleural }\end{array}$ & \multirow{2}{*}{$\begin{array}{c}G . \\
\text { adiacens } \\
G \text {. } \\
\text { adiacens }\end{array}$} & SR \\
\hline 14 & AML & none & & 0 & $\mathrm{~F}$, rash $\left(\mathrm{SJS} / \mathrm{TEN}^{5}\right)$ & VA IV $15 \mathrm{~d}$, CEP IV $4 \mathrm{~d}$ & & SR \\
\hline 15 & MDS & none & & 31 & $\begin{array}{l}F \text {, respiratory failure, } \\
\text { hypotension }\end{array}$ & $\begin{array}{l}\text { VA IV } 1 \mathrm{~d}, \text { CEP IV } 3 \mathrm{~d} \\
\text { DOX PO } 14 \mathrm{~d}\end{array}$ & $\begin{array}{c}\text { G. } \\
\text { adiacens }\end{array}$ & EX \\
\hline
\end{tabular}

AML: Acute Myeloid Leukemia, APML: Acute Promyelocytic Leukemia, ALL: Acute Lymphoblastic Leukemia, MDS: Myelodysplastic Syndrome, SJS/TEN: Stevens-Johnson/toxic epidermal necrolysis, NVS: Nutritionally Variant Streptococci, G. adiacens: Granulicatella adiacens, IV: Intravenous, PO: by mouth, MP: methylprednisolone, DXM: dexamethasone, CYT: Cytarabine, F: Fever, CFX: Ceftriaxone, VA: Vancomycin, PTZ: Piperacillin-Tazobactam, CEP: Cefepime, MER: Meropenem, ERT: Ertapenem, AMC: Amoxicillin-clavulanate, DOX: Doxycycline, LEV: Levofloxin, CIP: Ciprofloxacin, CEF: Ceftazidim, CEP: Cephalexin, CLN: Clindamycin, SR: Survived, EX: Deceased, n/a: data not available, 
Kwon J et al., Abiotrophia and Granulicatella Infections in Cancer Patients

Table 3. Characteristics of the patients with wound or body fluid aspirate positive cultures

\begin{tabular}{|c|c|c|c|c|c|c|c|}
\hline Case & Underlying Malignancy & Prior Procedures & Presentation/ Clinical Symptoms & Treatment & Source & Isolation & Outcome \\
\hline 1 & Cholangiocarcinoma & biliary stent exchange & decreased bilirubin & \multirow{11}{*}{$\begin{array}{c}\text { CFX IV4 } 2 \\
\text { PTZ IV } 1 \mathrm{~d} \\
\text { VA IV } 4 \mathrm{~d}, \text { CFX IV } 5 \\
\text { d, AMC PO } 9 \mathrm{~d} \\
\text { CEP IV } 3 \mathrm{~d}, \text { PTZ IV } \\
11 \mathrm{~d}, \text { LVQ PO } 7 \mathrm{~d} \\
\text { VA IV } 12 \mathrm{~d} \\
\text { CFD PO } 5 \mathrm{~d} \\
\text { CIP PO } 5 \mathrm{~d} \\
\text { SFT PO } 7 \mathrm{~d} \\
\text { catheter exchange } \\
\text { CEP IV } 3 \mathrm{~d} \\
\text { CFX IV } 2 \mathrm{~d} \\
\text { PTZ IV n/a } \\
\text { AMC PO } 14 \mathrm{~d} \\
\text { SFT PO } 14 \mathrm{~d} \text {, CIP } \\
\text { IV } 5 \mathrm{~d} \\
\text { VA IV } 2 \mathrm{~d}, \mathrm{PTZ} \text { IV } 4 \\
\text { d, LEV PO } 10 \mathrm{~d} \\
\text { CIP PO } 15 \mathrm{~d} \\
\text { CIP PO n/a } \\
\text { CHL mouth wash } \\
\text { CEP IV } 35 \mathrm{~d} \text {, CLN } \\
\text { IV } 28 \text { days, MER IV } \\
6 \mathrm{~d} \text {, CFD PO } 15 \mathrm{~d} \\
\text { VA IV } 2 \mathrm{~d} \text {, CEP IV } \\
12 \mathrm{~d}, \text { DAP IV } 1 \mathrm{~d} \text {, } \\
\text { Wound irrigation } \\
\text { debridement }\end{array}$} & body fluid aspirate (bile) & G. adiacens & SR \\
\hline 2 & Colon cancer & hemicolectomy & $\begin{array}{l}\text { fever, chills, abscess } \\
\text { (abdominal wall) }\end{array}$ & & $\begin{array}{l}\text { body fluid aspirate } \\
\text { (Abdominal abscess) }\end{array}$ & $N V S^{2}$ & SR \\
\hline 3 & $\begin{array}{l}\text { Adenocarcinoma } \\
\text { (esophagus) }\end{array}$ & $\begin{array}{l}\text { Ivor Lewis } \\
\text { esophagectomy }\end{array}$ & respiratory failure & & $\begin{array}{l}\text { body fluid aspirate } \\
\text { (pleural) }\end{array}$ & NVS & SR \\
\hline 4 & $\begin{array}{c}\text { Melanoma } \\
\text { (metastatic to lung) }\end{array}$ & thoracotomy & $\begin{array}{l}\text { Air leak from } \\
\text { thoracotomy chest tube }\end{array}$ & & $\begin{array}{l}\text { body fluid aspirate } \\
\text { (pleural) }\end{array}$ & NVS & SR \\
\hline 5 & Melanoma (left thigh) & $\begin{array}{l}\text { biopsy (left thigh } \\
\text { lesion) }\end{array}$ & cellulitis (left groin) & & $\begin{array}{l}\text { body fluid aspirate } \\
\text { (thigh seroma) }\end{array}$ & NVS & SR \\
\hline 6 & $\begin{array}{l}\text { Urothelial carcinoma, } \\
\text { Colon cancer }\end{array}$ & none & $\begin{array}{l}\text { lethargy, altered mental status, } \\
\text { nausea, vomiting, abdominal pain }\end{array}$ & & Urine & G. elegans & EX \\
\hline 7 & Colon cancer & colectomy (right) & pus from Wound site & & Wound (abdominal) & NVS & SR \\
\hline 8 & Breast cancer & breast implant & fever, cellulitis (left breast) & & $\begin{array}{l}\text { Wound (left breast } \\
\text { capsule) }\end{array}$ & G. adiacens & SR \\
\hline 9 & Lipoma (left thigh) & $\begin{array}{l}\text { left thigh drain } \\
\text { placement }\end{array}$ & $\begin{array}{l}\text { fever, decreased drain } \\
\text { output, swelling, redness }\end{array}$ & & Wound (left thigh) & G. adiacens & SR \\
\hline 10 & Acute myeloid leukemia & none & $\begin{array}{l}\text { fever, diarrhea, } \\
\text { molar cavity }\end{array}$ & & Wound (molar) & $\begin{array}{c}\text { A. } \\
\text { defectivus }\end{array}$ & SR \\
\hline 11 & $\begin{array}{l}\text { Osteosarcoma } \\
\text { (left thigh) }\end{array}$ & $\begin{array}{l}\text { distal femoral } \\
\text { replacement (left) }\end{array}$ & $\begin{array}{l}\text { hematoma and } \\
\text { quadriceps tear }\end{array}$ & & Wound (knee swab) & G. adiacens & SR \\
\hline 12 & $\begin{array}{l}\text { Gastrointestinal } \\
\text { stromal tumor }\end{array}$ & $\begin{array}{c}\text { flexible } \\
\text { sigmoidoscopy }\end{array}$ & GI bleed (melena) & None & Wound (pelvis swab) & G. adiacens & SR \\
\hline 13 & $\begin{array}{l}\text { Squamous cell carcinoma } \\
\text { (right lower lobe lung) }\end{array}$ & none & $\begin{array}{l}\text { secondary empyema of } \\
\text { right lung w/ abscess } \\
\text { fever, diarrhea }\end{array}$ & $\begin{array}{l}\text { CEP IV } 11 \mathrm{~d} \\
\text { TOB inhaled } 13 \mathrm{~d} \\
\text { CIP IV } 3 \mathrm{~d} \\
\text { LN IV n/a }\end{array}$ & Wound (pleural fluid) & NVS & SR \\
\hline
\end{tabular}

G. adiacens: Granulicatella adiacens, NVS: Nutritionally Variant Streptococci, G. elegans: Granulicatella elegans, A defectiva: Abiotrophia defective, IV: Intravenous, PO: by mouth, CFX: Ceftriaxone, VA: Vancomycin, CEP: Cefepime, MER: Meropenem, PTZ: Piperacillin-Tazobactam, AMC: Amoxicillin-clavulanate, SFT: sulfamethoxazole-trimethoprim, DOX: Doxycycline, LEV: Levofloxacin, CIP: Ciprofloxacin, LN: Linezolid, CEF: Ceftazidim, CEP: Cephalexin, CLN: Clindamycin, LVQ: Levaquin, TOB: Tobramycin, CFD: Cefdinir, CIP: Ciprofloxacin, DAP: Daptomycin, CHL: Chlorhexidine, SR: Survived 
Table 4. Most Common Antibiotic Regimen Used and Susceptibility Report.

\begin{tabular}{|c|c|c|c|c|c|}
\hline Antibiotic Treatment, n (\%) & Route, Duration & $\begin{array}{l}\text { Susceptibility } \\
\text { Report* }^{*}\end{array}$ & $\begin{array}{c}\text { Susceptible, } \\
n\end{array}$ & $\begin{array}{c}\text { Susceptible, } \\
n\end{array}$ & $\begin{array}{c}\text { Resistant, } \\
\mathrm{n}\end{array}$ \\
\hline VA, $18(64)$ & IV, 7.6 days & VA & 6 & & \\
\hline CEP, 13, (46) & IV, 6.8 days & MER, PEN G & 6 & 1 & \\
\hline PTZ, 6, (28) & IV, 4.2 days & CEP & 4 & 2 & \\
\hline \multirow[t]{4}{*}{ CFX, 4 (14) } & IV, 4 days & CFX & 3 & 1 & \\
\hline & & LEV & 1 & & 1 \\
\hline & & CEF & & 1 & \\
\hline & & CLN & & & 1 \\
\hline LEV 3, (11) & $\mathrm{PO}^{2}, 9.3$ days & ERT & & & 1 \\
\hline
\end{tabular}

Ceftriaxone, CEP: Cefepime, LEV: Levofloxacin, CEF: Ceftazidime, CLN: Clindamycin, ERT: Erythromycin

\section{DISCUSSION}

We collected the data of patients with positive Abiotrophia and Granulicatella cultures spanning a six-year period (Jan 2012-July 2018). Most Granulicatella and Abiotrophia infections present as bacteremia, which was consistent with our findings $[3,5]$. G. elegans has the lowest incidence in human specimens, as reflected in this study; their low infectivity is presumed to be due to its low frequency of colonization and low binding capacity [12-14].

Low granulocyte and neutrophil count, mucosal damage, and invasive procedures or foreign body material may increase the risk of infectious complications. Chemotherapy associated mucositis and neutropenia have been identified as risk factors for Granulicatella infections [8, 15-16]. Most patients with bacteremia had an underlying hematologic malignancy $(87 \%)$, and most of these patients were neutropenic for a significant amount of time (average 14.3 days). All but two patients with bacteremia were on cytotoxic chemotherapy agents or immunosuppressive agents as further listed in Table 2. Immune defects due to hematologic malignancies combined with immunosuppression from chemotherapy may put patients at increased risk for infectious complications. In addition, chemotherapy agents may induce mucositis or disrupt the integrity of mucosal lining, which may play a role in endogenous flora, such as NVS species, seeding into bloodstream [16].
Both A. defectiva and G. adiacens have shown high capacity to bind fibronectin and extracellular matrix proteins, which may contribute to their infectivity, especially in association with foreign body material [11]. These species have been reported in breast implants and hip arthroplasty [17,18]. The incidence of these species in association with foreign body material may rise due to the diagnostic improvements in molecular medicine and increasing use of foreign body material [1719]. Almost all patients in this study with wound or body fluid positive cultures had infections that were related to a site of a recent procedure or surgery. These findings suggest invasive procedures may predispose to Abiotrophia or Granulicatella infections in wound or body fluids, even in the absence of neutropenia or immunocompromised state. Symptoms for foreign body infections with these species are noted to present years later after implantation [17-19]. In contrast, patients in our study presented with symptoms within a much shorter period, an average of four months postprocedure. Regarding wound infections, appropriate use of antibiotics and removing any foreign body is reported to result in good clinical outcomes [17-19]. In our study, patients with infections related to foreign bodies recovered without recurrent infection after treatment with appropriate antibiotics and/or debridement of infected tissue and removal of the prosthesis.

Current guidelines do not reflect the susceptibility profiles of Abiotrophia and 
Granulicatella spp. For instance, the recommendations by the American Heart Association 2015 are that infective endocarditis caused by Granulicatella spp. be treated with a combination regimen of ampicillin or penicillin plus gentamicin [20]. However, according to Prasidthrathsint $\mathrm{K}$. et al, clinical isolates of $\mathrm{A}$. defectiva and $G$. adiacens have shown variable susceptibility to penicillin. A. defectiva was most susceptible to vancomycin, levofloxacin, ceftriaxone, clindamycin, and meropenem in vitro. G. adiacens showed similar susceptibility to vancomycin, levofloxacin, meropenem, but lower susceptibility to clindamycin and ceftriaxone [21]. These differences in susceptibility profiles may explain why positive cultures persisted in some patients despite prophylactic or prior empiric antibiotic therapy. The susceptibility reports in our study showed that vancomycin and piperacillin-tazobactam are useful agents for $\mathrm{G}$. adiacens. However, unlike susceptibility profiles reported in the literature, G. adiacens showed resistance to agents such as meropenem, ceftriaxone, and levofloxacin. The susceptibility report for A. defectiva was limited as there was only data available for one patient. Our findings demonstrate how these organisms can show variable susceptibility to broad-spectrum agents that are reported to be effective in the literature.

Twenty five (89\%) patients survived with no recurrent infections. Vancomycin IV was the most common treatment for these infections in our study, and most patients received multiple antimicrobial agents. Despite Granulicatella and Abiotrophia being sources of infective endocarditis reported in literature, we found no cases of endocarditis in this study, which may be related to the finding that only two patients had underlying heart conditions, neither of which had bacteremia. In addition, most patients (75\%) had prophylactic or prior empiric therapy prior or at the onset of symptoms, which may have precluded more serious infections. While no patients in this study needed central venous catheter removal, there is an increasing body of evidence that suggests that many gram-positive bacterial catheter infections can be successfully treated with the use of antimicrobial agents without catheter removal [22].

While Abiotrophia and Granulicatella isolates showed susceptibility to vancomycin in vitro, there are reports in literature where treatment failure was still observed [10]. The use of multiple antibiotic therapy in our study may have had a synergistic effect in preventing serious complications while effectively resolving Abiotrophia and Granulicatella infections. Three patients expired due to sepsis related complications. The single case of $G$. elegans was unsuccessfully treated with cefepime, ceftriaxone, and piperacillin-tazobactam, and susceptibility data was not available. However, these adverse outcomes cannot be attributed solely to treatment failure of Abiotrophia and Granulicatella, as these patients had polymicrobial infections.

Accurately identifying these species is important, especially in negative cultures. Phenotyping characterization is considered difficult due to the fastidious nature of these species, and conventional biochemical testing may lead to misidentification [2, 23].The presence of satellitism may be inconsistent, depending on the nutrients in the agar used, which can vary by manufacturer [9]. Although it is generally accepted that all three species grow well on chocolate agar or pyridoxal supplemented agar, there have been cases of $G$. elegans isolates only being supported by cysteine, and not pyridoxal or chocolate agar $[14,19]$. In a study comparing identification of various Abiotrophia and Granulicatella species using Vitek MS, Bruker MS, and Vitek 2, Vitek MS was the most superior, while the Vitek 2 system showed limitations to correct identification [24]. Especially with negative cultures, 16S rRNA sequencing was recommended for identification $[23,24]$. Even with improvements in culture media and speciation technology, there are still nuances and challenges to correctly identifying Abiotrophia and Granulicatella spp. which laboratories need to be aware.

\section{Limitations of Study}

Our study has several limitations. First, its retrospective design limited the available data on each patient. Second, we did not have adequate data to further analyze the differences between each species, as further speciation data was done only for patients with more severe clinical presentation or this information was not available in the patients' records. We were thus unable to further analyze an optimal 
treatment regimen for each species, as susceptibility reports and speciation data were limited. Finally, the use of Vitek 2 to further speciate NVS in this study may have contributed inaccuracies and limitations of this study despite resolution of infections in most patients. Using $16 S$ rRNA sequencing in the future may improve the accuracy of identifying the different species.

\section{Conclusion}

The difficulty of isolation, the potential misidentification, and serious pathogenicity associated with Granulicatella and Abiotrophia infections highlight how important it is for clinicians and laboratories to be aware of these infections. With the improvement of molecular diagnostics and identification, we can expect the incidence of Granulicatella and Abiotrophia infections to grow. Improved guidelines on antimicrobial therapy and continued surveillance are still needed for more effective treatment. While IV vancomycin has been shown in literature and our study to be the most effective agent, using multiple antibiotic regimens may have a synergistic effect that can more effectively resolve Abiotrophia and Granulicatella infections, especially if recommended guidelines do not lead to favorable outcomes. Clinicians need to be aware of the pathogenicity and presentation of these infections, especially in neutropenic patients or patients who have undergone recent procedures. This may be especially relevant in cancer patients, who may be more prone to immunosuppression and neutropenia with anticancer therapies, or invasive procedures related to solid tumor removal.

\section{ACKNOWLEDGMENTS}

All authors have participated significantly in writing of this manuscript and approve of its content. The final manuscript has been seen and approved by all authors.

Declaration of Conflicting Interests: The authors declare that they have no conflict of interest.

Financial Disclosure: No financial support was received.

\section{REFERENCES}

1. Frenkel A and Hirsch W. Spontaneous development of $\mathrm{L}$ forms of streptococci requiring secretions of other bacteria or sulfhydryl compounds for normal growth. Nature 1961; 191: 728-30.
2. Ruoff KL. Nutritionally variant streptococci. Clin Microbiol Rev 1991; 4: 184-90.

3. Christensen JJ and Facklam RR. Granulicatella and Abiotrophia species from human clinical specimens. J Clin Microbiol 2001; 39: 3520-3.

4. Ramos JN, dos Santos LS, Vidal LM, et al. A case report and literature overview: Abiotrophia defectiva aortic valve endocarditis in developing countries. Infection 2014; 42: 579-84.

5. Senn L, Entenza JM, Greub G, et al. Bloodstream and endovascular infections due to Abiotrophia defectiva and Granulicatella species. BMC Infect Dis 2006; 6: 9.

6. Zenone $T$ and Durand DV. Brain abscesses caused by Abiotrophia defectiva: complication of immunosuppressive therapy in a patient with connective-tissue disease. Scand J Infect Dis 2004; 36: 497-9.

7. Hepburn MJ, Fraser SL, Rennie TA, Singleton CM, Delgado B. Septic arthritis caused by Granulicatella adiacens: diagnosis by inoculation of synovial fluid into blood culture bottles. Rheumatol Int 2003; 23: 255-7.

8. Yacoub AT, Krishnan J, Acevedo IM, Halliday J, Greene JN. Nutritionally variant streptococci bacteremia in cancer patients: a retrospective study, 1999-2014. Mediterr J Hematol Infect Dis 2015; 7: e2015030.

9. Koh YR, Yi J, Kim HH, Chang CL, Kim SY. Discrepant Satellitism for Identification of Granulicatella adiacens Isolates. Annals of Laboratory Medicine 2014; 34: 174-176.

10. De Luca M, Amodio D, Chiurchiu S, et al. Granulicatella bacteraemia in children: two cases and review of the literature. BMC Pediat. 2013; 13: 61.

11. Okada Y, Kitada K, Takagaki M, Ito HO, and Inoue M. Endocardiac infectivity and binding to extracellular matrix proteins of oral Abiotrophia species. FEMS Immunology \& Medical Microbiology 2000; 27: 257-261.

12. Sato ST, Kanamoto T and Inoue M. Abiotrophia elegans strains comprise $8 \%$ of the nutritionally variant streptococci isolated from the human mouth. J Clin Microbiol 1999; 37: 2553-6.

13. Mvelase NR, Marajh $\mathrm{K}$, Hattingh $\mathrm{O}$, and Mlisana KP. An unusual case of thoracic empyema caused by Granulicatella elegans (nutritionally variant streptococci) in a patient with pulmonary tuberculosis and human immunodeficiency virus infection. JMM Case Rep 2016; 3: e005058.

14. Kanamaru A and Tatsumi Y. Microbiological Data for Patients with Febrile Neutropenia. Clinical Infectious Diseases 2004; 39: (S7-10).

15. Murray CK, Walter EA, Crawford S, McElmeel ML, and Jorgensen $\mathrm{JH}$. Abiotrophia bacteremia in a 
patient with neutropenic fever and antimicrobial susceptibility testing of Abiotrophia isolates. Clin Infect Dis 2001; 32: E140-2.

16. Sonis ST, Elting LS, Keefe D, et al. Perspectives on cancer therapy-induced mucosal injury. Cancer 100: 1995-2025. doi:10.1002/cncr.20162

17. del Pozo JL, Garcia-Quetglas E, Hernaez S, et al. Granulicatella adiacens breast implant-associated infection. Diagn Microbiol Infect Dis 2008; 6: 58-60.

18. Rozemeijer W, Jiya TU, Rijnsburger M, Heddema E, Savelkoul P, and Ang W. Abiotrophia defectiva infection of a total hip arthroplasty diagnosed by $16 S$ rRNA gene sequencing. Diagn Microbiol Infect Dis 2011 ; 70: $142-4$.

19. Casalta JP, Habib G, La Scola B, Drancourt M, Caus T, and Raoult, D. Molecular Diagnosis of Granulicatella elegans on the Cardiac Valve of a Patient with Culture-Negative Endocarditis. Journal of Clinical Microbiology 2002; 40: 1845-1847.

20. Baddour LM, Wilson WR, Bayer AS, et al. Infective Endocarditis in Adults: Diagnosis, Antimicrobial Therapy, and Management of Complications: A Scientific Statement for Healthcare Professionals From the American Heart Association. Circulation 2015; 132: 1435-86.

21. Prasidthrathsint $\mathrm{K}$, and Fisher MA. Antimicrobial Susceptibility Patterns among a Large, Nationwide Cohort of Abiotrophia and Granulicatella Clinical Isolates. J Clin Microbiol 2017; 55: 1025-1031.

22. O'Grady NP, Alexander M, Burns LA, et al. Summary of Recommendations: Guidelines for the Prevention of Intravascular Catheter-related Infections. Clinical Infectious Diseases 2011; 52: 1087-1099.

23. Cargill JS, Scott KS, Gascoyne-Binzi D, Sandoe JA. Granulicatella infection: diagnosis and management. J Med Microbiol 2012; 61(6): 755-61.

24. Ratcliffe $P$, Fang $H$, and Thidholm E. Comparison of MALDI-TOF MS and VITEK 2 System for Laboratory Diagnosis of Granulicatella and Abiotrophia Species Causing Invasive Infections. Diagnostic Microbiology and Infectious Disease 2013; 77: 216-219. 